\title{
Concentrador de Serviços de Telefonia Fixo e Móvel-CFM ${ }^{1}$
}

\author{
André L. Souza, Danilo Caravana, Eduardo M. Franceschini, George E. Bozinis, Guilherme J. de Souza, J. \\ Sindi Yamamoto e Rafael P. de A. Moreno
}

${ }^{1}$ Patente pendente

Resumo-- Neste trabalho, é apresentado o cenário atual de convergência fixo-móvel e as várias soluções tecnológicas para a sua implementação. Neste contexto, são também apresentadas as principais características, aplicações e os resultados de desempenho preliminares de uma femtocélula de baixo custo, para o usuário residencial ou corporativo de telefonia fixa e celular, chamado CFM (Concentrador de Serviços de Telefonia Fixo e Móvel). O CFM, desenvolvido pela Innovatech Telecom, promove a convergência fixo-móvel de forma muito simples para os usuários e da forma mais otimizada possível para as operadoras, pois, aos usuários permite acessar a rede fixa do seu próprio terminal celular GSM e, para as operadoras, não há necessidade de empregar novas tecnologias de elementos de rede.

Palavras chaves-- convergência fixo-móvel, femto-célula, CFM

\begin{abstract}
In this work, the current fixed-mobile scene and several technological solutions for its implementation are presented. In this context, the main characteristics, applications and the preliminary performance results of a low cost femtocell, for the residential or corporative users of fixed and cellular telephony, called CFM (Fixed-Mobile Services Concentrator), are also presented. The CFM, developed by the Innovatech Telecom, promotes the fixedmobile convergence in a very simple way for the users and in the most possible optimized way for the operators, since it allows the users to have access to the fixed network from their own GSM cellular terminal and without the necessity of using new network elements technologies on the part of the operators.
\end{abstract}

Key words-- fixed-mobile convergence, femtocell, CFM

\section{INTRODUÇÃO}

A questão sobre a convergência de sistemas de telefonia

André L. Souza, Danilo Caravana, Eduardo M. Franceschini, George E. Bozinis, Guilherme J. de Souza, J. Sindi Yamamoto e Rafael P. de A. Moreno Innovatech Telecom, Galleria Office Park: Av. Selma Parada, 201-Bloco 3 Sala 301, Jardim Madalena - Campinas - SP http://www.innovatechtelecom.com

e-mail: innovatech@innovatechtelecom.com

Este trabalho foi parcialmente financiado pela FAPESP (Proc. no. 03/13148-4) fixa e móvel tem atraído uma atenção crescente diante da evolução tecnológica e a constante evolução das redes para uma estrutura baseada em rede IP. Recentemente, importantes operadoras de telefonia formaram uma associação e compuseram o FMCA ("Fixed Mobile Convergence Association”) [1] com o objetivo de estimular a adoção de soluções tecnológicas que possibilitem a implantação da convergência fixo-móvel.

No núcleo da rede, tecnologias como o IMS ("IP Multimedia Subsystem") [2] e UMA ("Unlicensed Mobile Alliance”) [3] representam importantes contribuições para a convergência fixo-móvel. O IMS é definido pelo 3GPP/3GPP2 como uma plataforma arquitetural que permite a convergência das tecnologias de dados, voz e rede sobre uma infra-estrutura baseada em IP. Para a operadora, representa uma opção para controle e lógica de serviço para comunicação fim a fim baseado em pacotes IP. Para os usuários, serviços baseados em IMS permitirão comunicações em vários modos, incluindose voz, texto, fotos e vídeo, ou qualquer combinação destes, de uma forma pessoal e segura.

A figura 1 ilustra o conceito da UMA. A especificação é exclusiva para redes GSM/GPRS e utiliza a infra-estrutura de comutação da rede GSM. A UMA permite que chamadas de voz e dados sejam realizadas através da rede IP fixa desviando a carga de tráfego da rede GSM para uma rede IP de maior largura de banda por um custo menor. A rede IP fixa é acessada através de pontos de acesso, AP ("Access Point”) WiFi ou Bluetooth. Para o "core” da rede móvel celular, os AP são como um outro terminal móvel qualquer conectado através da BSC ("Base Station Controller"). O problema da UMA é que o mesmo opera somente com terminais dual mode celular/WiFi ou Bluetooth com certificação UMA. Além disto, um terminal UMA não é bem recebido pelas operadoras, pois tem-se o receio de acesso irrestrito aos APs WiFi e, assim, os usuários realizarem “download” de aplicações, como o Skype, e não necessitarem mais da operadora. Além disto, a UMA necessita de instalação de um novo elemento de rede: UNC (“Universal Network Controller”).

Já na rede de acesso, existe uma grande quantidade de tecnologias que se apresentam como opções importantes para a interconexão das instalações do assinante com a rede IP. Além de tecnologias de acesso já existentes, têm-se as novas tecnologias de acesso de banda larga sem fio e os sistemas de terceira geração da ITU-T, denominados IMT-2000 [4]. 
Entretanto, estas tecnologias precisam ser integradas de alguma forma para promover a convergência da rede de telefonia fixa e móvel.

Como resultado dos esforços do FMCA, liderado pela British Telecom (BT) com o seu projeto original, denominado Bluephone, e lançado comercialmente com o nome BT Fusion [5], a convergência fixo-móvel ganhou um novo patamar em sua escalada. Em uma primeira fase, um sistema com acesso fixo baseado em Bluetooth [6] foi especificado pelo FMCA. Entretanto, devido às limitações impostas pela interface Bluetooth e sua susceptibilidade às interferências, em uma nova fase do sistema, o mesmo deverá evoluir para uma interface Wi-Fi [7].

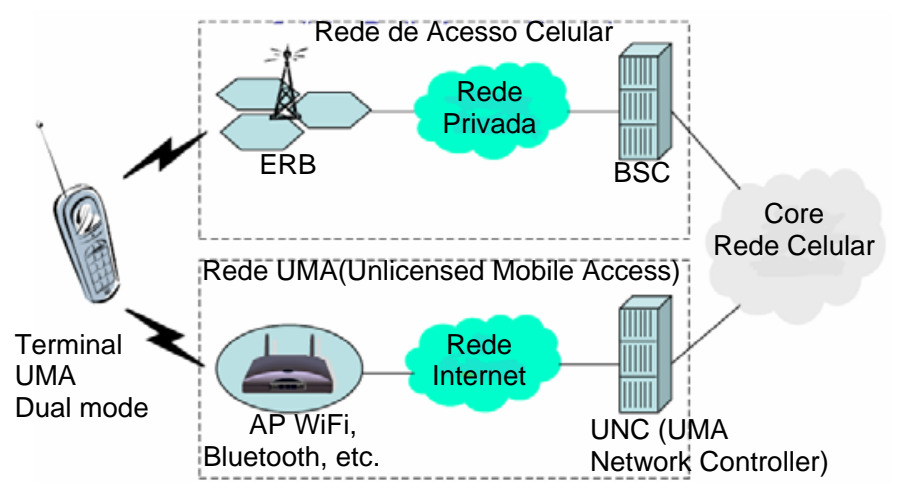

Fig.1. Conceito da UMA

Todas estas soluções exigem a utilização de terminais celulares "dual mode" WiFi ou Bluetooth com certificação UMA, e de um novo elemento de rede (UNC). Entende-se que o maior obstáculo para o sucesso destas soluções de convergência fixo-móvel não está no núcleo da rede, mas na disponibilidade e utilização de terminais celulares dual mode WiFi ou Bluetooth. A tecnologia Bluetooth já se encontra disponível em terminais celulares "high end" GSM e CDMA há algum tempo, de forma que pode ser considerada uma tecnologia madura. Mas, o Bluetooth, como já mencionado, é muito susceptível à interferências [8] e tem alcance limitado. Já em relação aos terminais celulares/WiFi, existem atualmente poucos modelos e os mesmos apresentam alto consumo, de forma que o tempo de duração das baterias sem recarga é muito baixa quando comparado com a de terminais celulares sem WiFi.

A Innovatech Telecom tem focado no desenvolvimento de uma tecnologia de convergência fixo-móvel que contorna todos os problemas mencionados acima. Nesta linha, desenvolveu uma femto-célula chamada CFM (Concentrador de Serviços de Telefonia Fixo e Móvel), que promove a convergência fixo-móvel utilizando-se quaisquer terminais celulares GSM e sem a necessidade de novos elementos de rede. Assim, o CFM permite implementar a convergência fixomóvel de uma forma muito simples para o usuário e da forma mais otimizada possível para a operadora.
Neste trabalho, são apresentadas as principais características, aplicações e alguns resultados de desempenho do CFM. Para tanto, o trabalho encontra-se organizado da seguinte forma: inicialmente, na seção II é apresentado o conceito do CFM. Na seção III são descritas as principais características e funcionalidades do CFM, bem como os requisitos de rede das operadoras para a sua operação. Na seção IV são apresentadas algumas das aplicações e principais benefícios do CFM para os usuários e operadoras. Finalmente, resultados de medidas de desempenho realizadas $\mathrm{e}$ as principais conclusões são apresentados nas seções V e VI, respectivamente.

\section{CONCEITO DO CFM}

O CFM é uma femto-célula GSM que permite a integração dos serviços da rede fixa com os serviços da rede móvel celular GSM. A figura 2 ilustra o conceito do CFM. Como mostrado, o CFM possui, de um lado, interface sem fio compatível com o padrão GSM, permitindo, desta forma, que um terminal móvel celular GSM inter-opere com o mesmo como se fosse uma ERB (Estação Rádio Base). Do outro lado, o CFM possui interfaces que permitem a inter-operação com a rede fixa comutada, seja através da linha telefônica analógica ou algum equipamento de banda-larga como o ADSL.

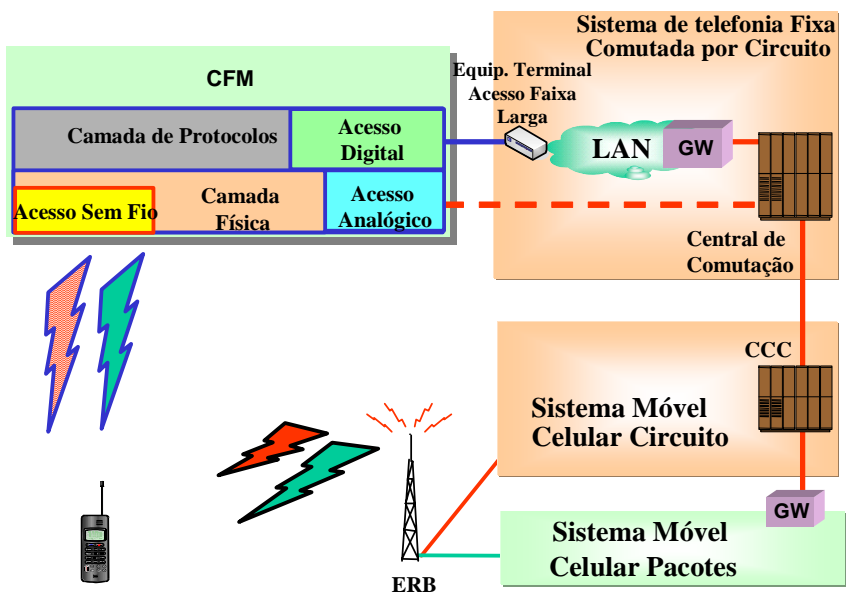

Fig. 2. Conceito do CFM

$\mathrm{O}$ acesso à rede fixa comutada através da linha telefônica analógica é realizado através de uma interface de acesso analógico, representada na figura 2 pela linha pontilhada. Para este caso de acesso, o CFM converte o sinal de voz codificado à 13 kbps, segundo o padrão GSM 06.60 ("Enhanced Full Rate-EFR-Speech Transcoding”), recebido através da interface rádio, para um sinal de voz analógico. Inicialmente, o sinal de voz à 13 kbps é decodificado para PCM linear de 13 bits na camada física. O sinal PCM é convertido para o domínio analógico na interface de acesso analógico. Do mesmo modo, o sinal de voz analógico proveniente da linha telefônica através da interface de acesso analógico, é inicialmente codificado em PCM linear de 13 bits. O sinal PCM é depois transcodificado à 13 kbps na camada física e transmitido através da interface 
rádio compatível com o padrão GSM. A sinalização entre o CFM e a rede telefônica consiste de sinalização de assinante gerado pela camada de protocolos e interface de acesso analógico.

$\mathrm{O}$ acesso à rede fixa através de equipamento de banda-larga como, por exemplo, ADSL, é realizado pela interface de acesso digital. Neste caso, o sinal de voz é codificado à 13 kbps segundo o padrão GSM 06.10 ("Full Rate (FR) speech transcoding”). O sinal de voz à 13 kbps é convertido em pacotes IP na interface de acesso digital e transmitido abrindose uma sessão VoIP ("Voice over IP") sobre uma conexão UDP/IP. Adicionalmente, a interface digital utiliza o protocolo SIP("Session Initiation Protocol”) [9] para o estabelecimento de uma sessão VoIP e o protocolo RTP (“Real Time Protocol”) para a transferência de mídia [10].

Além das funções já mencionadas, a camada de protocolos do CFM implementa parte das camadas $2 / 3$ da pilha de protocolos GSM do lado da rede celular necessária para a realização de acampamento, re-seleção de células, originação/terminação de chamadas [11,12,13] e envio/recebimento de SMS ("Short Message Services”) $[14,15]$ utilizando-se o protocolo SMPP v.3.4 [16].

Comparando-se o conceito do CFM com o da UMA, a grande diferença é que o CFM baseia-se na utilização de quaisquer terminais móveis GSM já em uso pelos usuários bem como de elementos de rede já existentes e consagrados na rede telefônica. Assim, ao contrário das outras tecnologias, o sucesso do CFM não depende de novos terminais móveis e nem de novos elementos de rede.

\section{Principais Características do CFM}

O CFM permite a convergência fixo-móvel de forma mais simples para o usuário e otimizada para a operadora, pois permite a um usuário, com qualquer terminal celular GSM, realizar ou receber ligações através da rede fixa, e a operadora não necessita instalar nenhum novo elemento de rede além dos já instalados para comutação por circuitos e VoIP. Quando o usuário está localizado na área de cobertura do CFM (área residencial ou comercial “indoor”), o mesmo permite que o usuário acesse a rede fixa do seu próprio terminal celular GSM e se beneficie, por exemplo, da tarifa de rede fixa. Para tanto, o CFM consiste de uma femto-célula de baixo custo para o usuário residencial ou corporativo de telefonia fixa e celular, e foi desenvolvido em duas versões:

- Com interface de linha analógica: o CFM tem interface analógica com a rede fixa e permite que diversos terminais GSM estejam habilitados a efetuar e receber chamadas através do mesmo. Entretanto, devido às limitações da linha da rede fixa, somente uma única ligação pode ser realizada por vez.

- Com interface banda-larga: o CFM tem interface digital com a rede fixa através de um acesso de banda larga, e também permite que vários terminais GSM sejam habilitados a utilizar um único dispositivo CFM. Nesta versão, até quatro chamadas podem ser realizadas simultaneamente, por diferentes terminais GSM, utilizando a mesma linha da rede fixa. As chamadas de cada terminal móvel correspondem a diferentes sessões de chamadas VoIP ("Voice over IP") na rede fixa sob total controle da operadora fixa (ou provedora de VoIP) e/ou operadora móvel.

O terminal móvel do usuário, ao entrar na área de cobertura do CFM, seleciona a célula do CFM para acampamento baseado na prioridade do H-PLMN ("Home-Public Land Mobile Network") [17], transmitida pela portadora BCCH ("Broadcast Control Channel") do CFM, ou baseado no parâmetro de seleção de célula C2 [18]. No primeiro caso, o CFM e os usuários autorizados a acampar no CFM possuem uma PLMN diferente da operadora celular em cuja região de atendimento o CFM encontra-se instalado. Este é o caso de aplicação do CFM onde uma operadora celular deseja estender a sua cobertura a alguns usuários específicos fora da sua área de cobertura normal. No segundo caso, o CFM e os usuários autorizados utilizam a mesma PLMN da operadora celular em cuja área de atendimento o CFM encontra-se instalado.

Além das características acima, ambas as versões do CFM apresentam as seguintes principais características:

- Seleção automática de melhor freqüência para a portadora BCCH.

- Criação automática de usuários autorizados para uso do CFM a partir do próprio SimCard do usuário.

- Autenticação.

- Criptografia.

- Originação de SMS.

- Terminação de SMS.

- “Calling Line Identification” (Identificador de chamadas).

\section{III.1. REQUISITOS DA OPERADORA}

\section{a. CFM com Interface Analógica}

No caso de utilização do CFM com interface analógica, são utilizados os elementos de rede já existentes para comutação de circuitos tanto na rede móvel como na rede fixa. Quando o terminal móvel estiver acampado no CFM, e portando fora da cobertura da rede celular, a MSC da operadora móvel deve redirecionar a chamada para o número da linha telefônica fixa onde o CFM estiver conectado. Assim, a operadora móvel deve ativar o serviço suplementar "Call Forwarding on User Not Reachable”.

\section{b. CFM com Interface Digital}

Na figura 3, são mostrados os elementos de rede envolvidos na operação do CFM com interface digital. Observa-se que são utilizados os elementos de rede já existentes para a comutação por circuitos e comutação por pacotes VoIP baseados no 
protocolo SIP. Desta forma, se a operadora já provê o serviço de VoIP baseado no protocolo SIP, não é necessária a instalação de novos elementos de rede. Neste caso, cada terminal móvel acampado no CFM deve possuir um segundo número, denominado número fixo do usuário, devidamente cadastrado no servidor Proxy SIP da operadora. Assim, quando o terminal móvel estiver acampado no CFM, a MSC da operadora móvel redireciona a chamada para o número fixo do usuário através do serviço suplementar "Call Forwarding on User Not Reachable”. A central de comutação, ao receber uma chamada com este número fixo, encaminha a chamada para a media gateway. A media gateway encaminha a chamada para o servidor SIP ("SIP server") que abre uma conexão UDP/IP com o CFM e uma sessão VoIP sobre esta conexão correspondente ao número fixo do usuário.

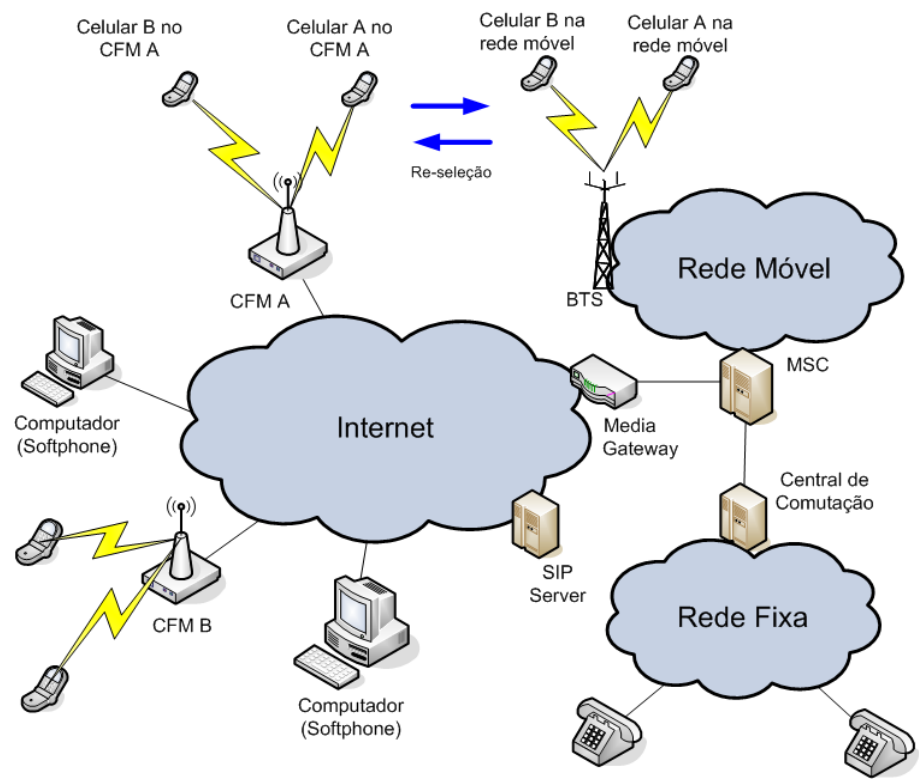

Fig.3. Elementos de rede envolvidos na operação do CFM com interface digital

\section{APLICAÇões E BENEFÍ́cios}

O CFM, além de promover a convergência fixo-móvel que otimiza a utilização da infra-estrutura e recursos de rede, permite a realização de diversas aplicações à um custo extremamente baixo para as empresas operadoras, pois não há necessidade de investimentos em infra-estrutura de rede. Ao contrário, o CFM permite uma forte redução de investimentos em capacidade de rede e uma otimização de todos os investimentos já realizados. Como alguns exemplos de aplicação do CFM, podem ser citados: a melhoria de cobertura "indoor", a expansão de cobertura para atendimentos específicos, residenciais ou corporativos, sem investimentos adicionais e a solução de problemas de transmissão e de cobertura de locais onde a sua implementação são inviáveis economicamente através de métodos convencionais.

Em termos de benefícios para a operadora e usuários, podem ser citados: a) Benefícios para os usuários: economia com menores tarifas quando estiver na área de cobertura do CFM; melhoria da qualidade de serviço “indoor”; encaminhamento das chamadas provenientes das redes fixas e móveis, para um único terminal GSM, facilitando o gerenciamento de vários acessos e reduzindo a perda de chamadas; conforto com a utilização de um terminal móvel.

b) Benefícios para a operadora: redução de investimentos em capacidade de rede; fidelização de clientes (redução de “churn”), maior flexibilidade de expansão de cobertura; aumento da receita com maior disponibilidade de serviços; solução de diversos problemas de cobertura e de transmissão.

\section{DESEMPENHO}

Em termos de desempenho do CFM, são importantes a qualidade de voz, o tempo de re-seleção da célula CFM e a sua cobertura. Devido às condições mais favoráveis de propagação “indoor” quando comparado com as condições de propagação "outdoor", testes subjetivos informais mostram uma boa qualidade de voz quando as ligações telefônicas são realizadas através do CFM via interface de acesso analógica ou bandalarga. Em termos de re-seleção de célula, conforme descrito na seção III, o CFM permite a re-seleção da sua célula baseado na prioridade da H-PLMN ou no parâmetro C2. No primeiro caso, o tempo de re-seleção depende do valor $\mathrm{T}$ minutos programado no SimCard do usuário. O valor de T encontra-se na faixa de 6 minutos a 8 horas, em passos de 6 minutos, ou ainda pode indicar que nenhuma tentativa periódica deve ser feita. Se nenhum valor é armazenado, um valor de T igual a 30 minutos é utilizado. Assim, para uma programação de $\mathrm{T}=6$ minutos, a primeira seleção da célula CFM, isto é, quando o terminal móvel é ligado, ocorre entre um tempo mínimo de 2 minutos e no máximo de 6 minutos. As re-seleções subsequentes da célula do CFM ocorrem em um intervalo de tempo menor do que 6 minutos.

No caso de re-seleção da célula CFM baseado no parâmetro C2, a re-seleção da célula CFM ocorre em um intervalo de tempo menor do que 36 segundos.

As figuras 4 e 5 ilustram os ambientes corporativo $\left(\sim 289 \mathrm{~m}^{2}\right)$ e residencial $\left(\sim 82,5 \mathrm{~m}^{2}\right)$, respectivamente, onde foram realizadas as medidas de cobertura do CFM. Como pode ser observado, os ambientes foram divididos em pequenas células ( $\mathrm{Y}, \mathrm{G}$ e $\mathrm{B}$ ) de medidas. $\mathrm{O}$ ambiente coprporativo foi dividido em 69 células de medidas de $4 \mathrm{~m}^{2}$ : Y1 a Y23, G1 a G23 e B1 a B23. Da mesma forma, o ambiente residencial foi dividido em 58 células de medidas de aproximadamente 1,4 $\mathrm{m}^{2}$ : Y1 a Y19, G1 a G20 e B1 a B19. Os resultados das medidas em cada uma destas células, para uma potência de transmissão fixa no $\mathrm{CFM}$ de $0 \mathrm{dBm}$, são apresentados nas tabelas I e II para os ambientes corporativo e residencial, 
respectivamente. Observa-se que a potência da portadora BCCH do CFM (potência CFM) está, aproximadamente, na mesma faixa de valores da potência da portadora da BTS servidora da rede celular (potência BTS) em cada uma das celulas de medida. Entretanto, o valor do parâmetro C2 do CFM (C2 CFM) é sempre maior do que o da BTS servidora da rede celular (C2 BTS) em todas as células de medida. Assim, o terminal móvel manteve-se acampado no CFM em todas a células de medida nestes dois ambientes.

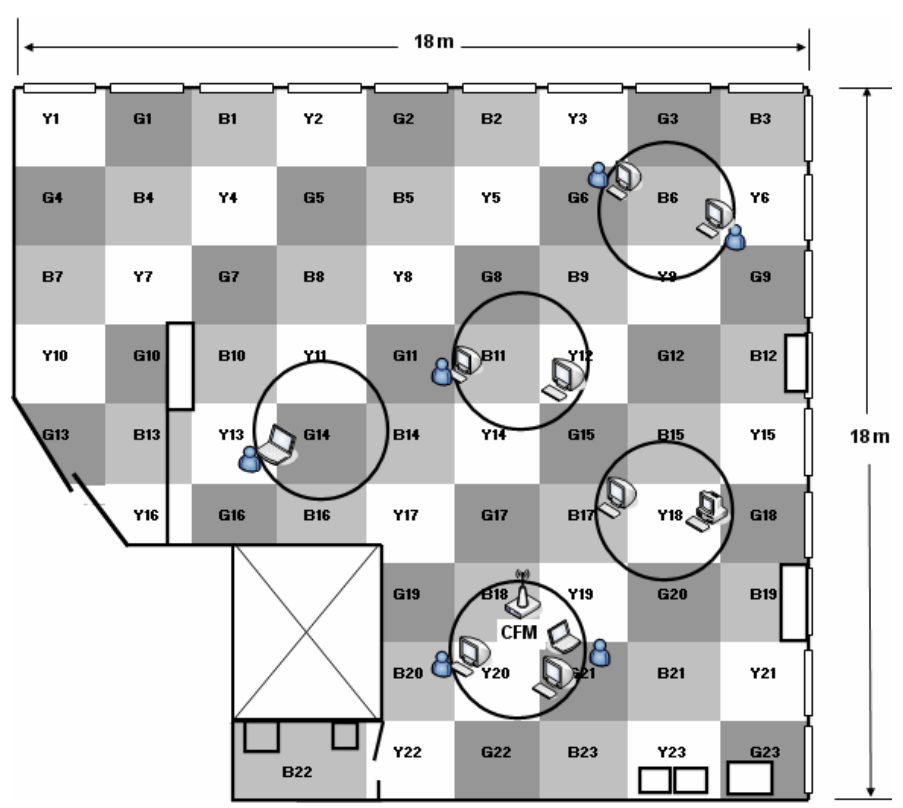

Fig. 4. Ambiente "indoor" corporativo.

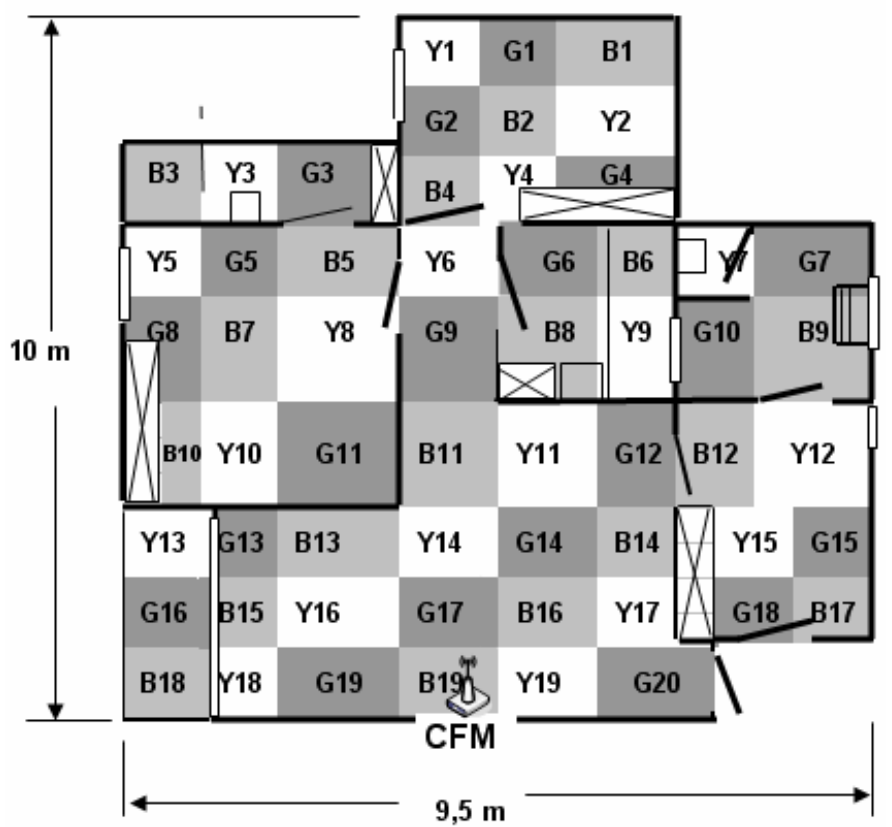

Fig. 5. Ambiente “indoor" residencial.

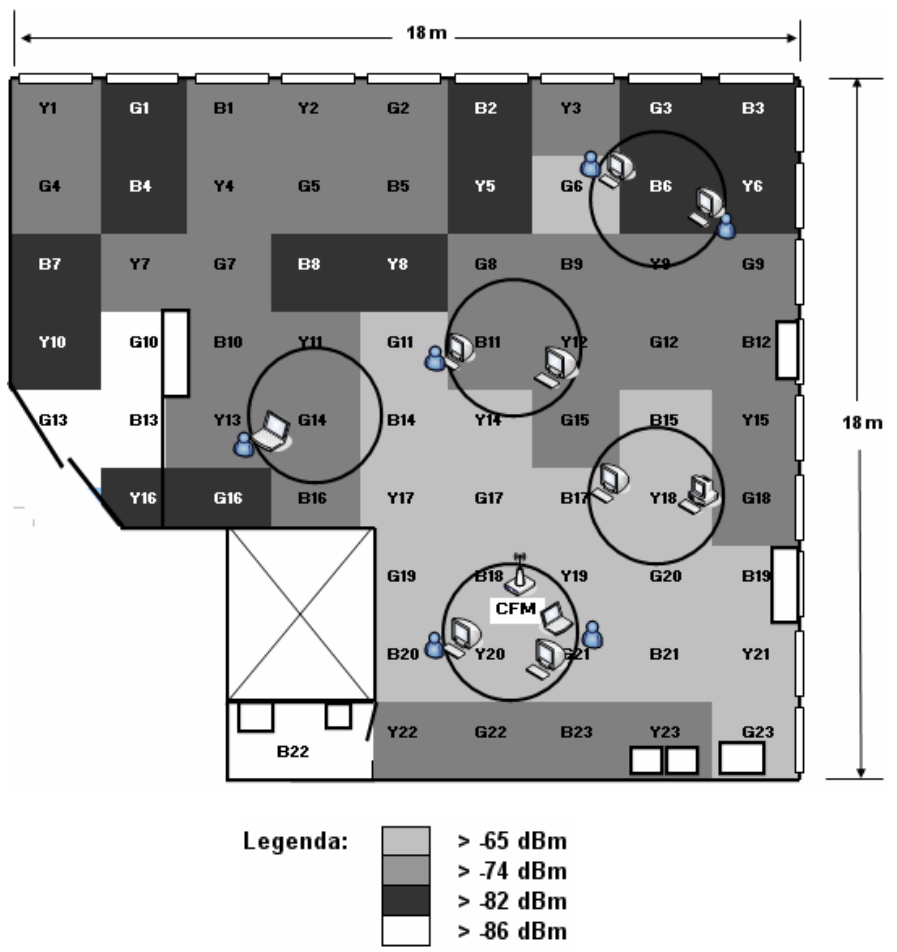

Fig.6. Cobertura do ambiente corporativo.

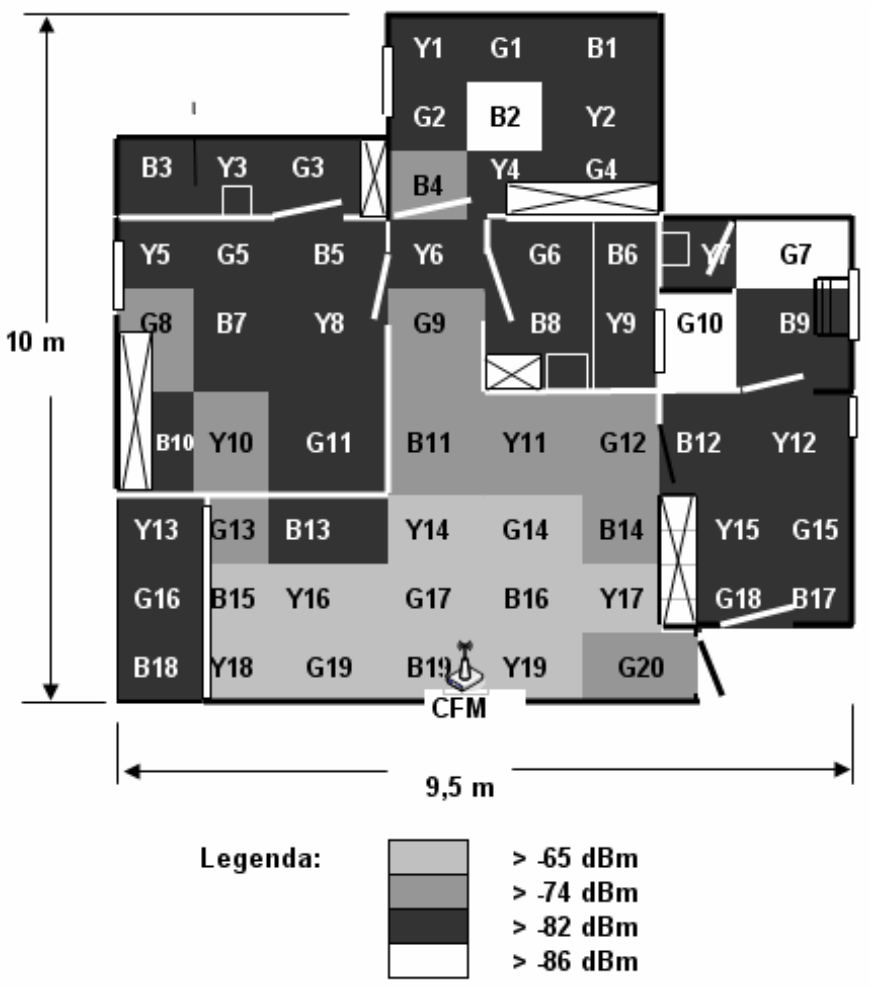

Fig.7. Cobertura do ambiente residencial. 
TABELA I

MedidAs de PotênCIA E C2 NO AMBIENTE CoRporativo

\begin{tabular}{|c|c|c|c|c|c|c|c|c|c|c|c|c|}
\hline \multirow{3}{*}{$\begin{array}{c}\text { Célula } \\
\text { no. }\end{array}$} & \multicolumn{4}{|c|}{$Y$} & \multicolumn{4}{|c|}{$G$} & \multicolumn{4}{|c|}{ B } \\
\hline & C2 BTS & Potênci & C2 CFM & Poténcia & $\mathrm{C} 2 \mathrm{BTS}$ & Potência & C2 CFM & Potência & C2 BTS & Poténc & C2 CFM & Potência \\
\hline & & BTS & & & & BTS & & CFM & & BTS & & CFM \\
\hline 1 & 28 & -75 & 49 & 66 & 26 & -77 & 37 & -78 & 36 & -67 & 45 & -70 \\
\hline 2 & 35 & -68 & 44 & -71 & 23 & -80 & 44 & -71 & 30 & -73 & 40 & -75 \\
\hline 3 & 29 & -74 & 42 & -73 & 29 & -74 & 35 & -80 & 25 & -78 & 40 & -75 \\
\hline 4 & 18 & -85 & 45 & -70 & 23 & -80 & 43 & -72 & 26 & -83 & 40 & -75 \\
\hline 5 & 29 & -74 & 40 & -75 & 27 & -76 & 46 & -69 & 22 & -81 & 47 & 68 \\
\hline 6 & 31 & -72 & 39 & -76 & 30 & -73 & 52 & -63 & 34 & -68 & 36 & -79 \\
\hline 7 & 31 & -72 & 45 & -70 & 30 & -73 & 46 & 69 & 25 & -78 & 41 & -74 \\
\hline 8 & 30 & -73 & 40 & -75 & 22 & -81 & 50 & -65 & 24 & -79 & 39 & -76 \\
\hline 9 & 36 & 67 & 43 & -72 & 38 & -65 & 51 & -64 & 25 & -78 & 42 & -73 \\
\hline 10 & 33 & -70 & 39 & -76 & 23 & -80 & 29 & -86 & 31 & -72 & 51 & -64 \\
\hline 11 & 29 & -74 & 44 & -71 & 39 & 64 & 52 & 63 & 31 & -72 & 49 & -66 \\
\hline 12 & 33 & -70 & 48 & 67 & 31 & -72 & 49 & -66 & 34 & 69 & 51 & -64 \\
\hline 13 & 24 & -79 & 46 & 69 & 32 & -71 & 32 & -83 & 15 & -77 & 51 & 64 \\
\hline 14 & 26 & -77 & 55 & 60 & 40 & 63 & 48 & -67 & 34 & 69 & 57 & -58 \\
\hline 15 & 33 & -70 & 42 & -73 & 36 & -67 & 47 & -68 & 41 & 62 & 52 & -63 \\
\hline 16 & 39 & -64 & 36 & -79 & 29 & -74 & 41 & -74 & 31 & -72 & 44 & -71 \\
\hline 17 & 32 & -71 & 52 & 63 & 39 & -68 & 51 & -64 & 39 & -64 & 58 & -57 \\
\hline 18 & 39 & 64 & 55 & 60 & 37 & 66 & 51 & -64 & 31 & -72 & 48 & -67 \\
\hline 19 & 34 & 69 & 61 & -54 & 36 & -67 & 52 & -63 & 23 & -80 & 52 & -63 \\
\hline 20 & 26 & -77 & 52 & -63 & 33 & -70 & 51 & -64 & 31 & -72 & 52 & -63 \\
\hline 21 & 27 & -76 & 51 & 64 & 29 & -74 & 52 & -63 & 31 & -72 & 53 & -62 \\
\hline 22 & 21 & -82 & 42 & -73 & 30 & -75 & 45 & -70 & 19 & -84 & 30 & -85 \\
\hline 23 & 35 & -66 & 49 & 66 & 30 & -73 & 55 & 60 & 33 & -70 & 50 & 65 \\
\hline
\end{tabular}

TABELA II

MEdidAs DE PotÊnCIA E C2 NO AMBIENTE RESIDENCIAL

\begin{tabular}{|c|c|c|c|c|c|c|c|c|c|c|c|c|}
\hline \multirow{2}{*}{\begin{tabular}{|c|} 
Célula \\
no.
\end{tabular}} & \multicolumn{4}{|c|}{$Y$} & \multicolumn{4}{|c|}{ G } & \multicolumn{4}{|c|}{ B } \\
\hline & BTS & Potência & C2 CFM & Potência & C2 BTS & Potència & $\begin{array}{l}a \\
\end{array}$ & Potência & 2 BTS & Poténcia & C2 CFM & Potência \\
\hline 1 & 15 & -88 & 40 & -75 & 19 & -84 & 34 & -81 & 24 & $\begin{array}{l}-79 \\
\end{array}$ & 36 & -79 \\
\hline 2 & 18 & -85 & 34 & -81 & 14 & -89 & 39 & -76 & 18 & -85 & 31 & -84 \\
\hline 3 & 27 & -76 & 38 & -77 & 18 & -85 & 34 & -81 & 28 & -75 & 39 & -76 \\
\hline 4 & 13 & -90 & 35 & -80 & 23 & -80 & 36 & -79 & 21 & -82 & 44 & -71 \\
\hline 5 & 22 & -81 & 37 & -78 & 25 & -78 & 36 & -79 & 18 & -85 & 37 & -78 \\
\hline 6 & 21 & -82 & 37 & -78 & 16 & -87 & 36 & -79 & 21 & -82 & 35 & -80 \\
\hline 7 & 21 & -82 & 36 & $\begin{array}{l}-79 \\
\end{array}$ & 27 & -76 & 32 & -83 & 27 & -76 & 38 & -77 \\
\hline 8 & 17 & -86 & 35 & -80 & 23 & -80 & 43 & -72 & 16 & -87 & 36 & -79 \\
\hline 9 & 21 & -82 & 35 & -80 & 23 & -80 & 48 & -67 & 27 & -76 & 32 & -83 \\
\hline 10 & 27 & -76 & 45 & -70 & 21 & -82 & 32 & -83 & 26 & -77 & 35 & -80 \\
\hline 11 & 16 & -87 & 43 & -72 & 18 & -85 & 35 & -80 & 28 & -75 & 49 & -66 \\
\hline 12 & 17 & -86 & 40 & -75 & 20 & -83 & 45 & -70 & 21 & -82 & 37 & -78 \\
\hline 13 & 28 & -75 & 37 & -78 & 24 & -79 & 45 & -70 & 23 & -80 & 41 & -74 \\
\hline 14 & 18 & -85 & 51 & -64 & 22 & -81 & 51 & -64 & 24 & -79 & 48 & -67 \\
\hline 15 & 13 & -90 & 36 & -79 & 17 & -86 & 36 & -79 & 28 & -75 & 51 & -64 \\
\hline 16 & 21 & -82 & 58 & -57 & 30 & -73 & 38 & -77 & 27 & -76 & 56 & -59 \\
\hline 17 & 21 & -82 & 52 & 63 & 28 & -75 & 57 & -58 & 21 & -82 & 34 & -81 \\
\hline 18 & 24 & -79 & 54 & -61 & 8 & -95 & 40 & -75 & 25 & -78 & 36 & -79 \\
\hline 19 & 18 & -85 & 53 & -62 & 28 & -75 & 55 & 60 & 22 & -81 & 74 & -41 \\
\hline 20 & & & & & 24 & -79 & 50 & -65 & & & & \\
\hline
\end{tabular}

Nomenclatura:

- C2 BTS: parâmetro C2 de uma BTS servidora da rede celular calculado pelo terminal móvel;

- C2 CMF: parâmetro C2 do CFM calculado pelo terminal móvel;

- Potência BTS: potência da portadora BCCH de uma BTS servidora da rede celular medida pelo terminal móvel;

- Potência CFM: potência da portadora BCCH do CFM medida pelo terminal móvel.

As figuras 6 e 7 ilustram as coberturas obtidas para os ambientes corporativo e residencial, respectivamente. No caso do ambiente corporativo, obteve-se que $27,7 \%$ da área é coberta com potência superior a $-65 \mathrm{dBm}$ (área próxima ao CFM). As outras porcentagens de cobertura são: potência entre $-74 \mathrm{dBm}$ e $-65 \mathrm{dBm}$ : 46,8\%; potência entre $-82 \mathrm{dBm}$ e -74 $\mathrm{dBm}$ : 20,6\% e potência entre $-86 \mathrm{dBm}$ e $-82 \mathrm{dBm}: 4,8 \%$.

No caso do ambiente residencial, a área mais próxima do CFM tem potência acima de -65 dBm e corresponde a 19\% da área total. As outras porcentagens de cobertura são: potência entre -74 dBm e -65 dBm: 17,2\%; potência entre -82 dBm e $74 \mathrm{dBm}$ : 58,6\% e potência entre $-86 \mathrm{dBm}$ e $-82 \mathrm{dBm}$ : 5,2\%.

\section{CONCLUSÕES}

Neste trabalho foram apresentadas as diversas soluções de tecnologias propostas para a convergência fixo-móvel e os problemas associados para o sucesso destas tecnologias. Em particular, o CFM apresenta a vantagem de não necessitar de novos terminais móveis ou terminais móveis específicos e nem de uma nova tecnologia de elemento de rede, facilitando, assim, a sua implementação por parte das operadoras e seu acesso e utilização pelos usuários. Adicionalmente, os resultados de testes de desempenho, até então obtidos, têm demonstrado o seu potêncial de atendimento às aplicações e benefícios descritos no trabalho.

\section{REFERÊNCIAS}

[1] http://www.thefmca.com/

[2] GPP TS 22.228 "Service requirements for the Internet Protocol (IP) multimedia core network subsystem (IMS); Stage 1" v7.2.0; 6/10/2005

[3] UMA User Perspective - Stage 1; R.1.0.0; 1/09/2004.

[4] http://www.itu.int/home/imt.html

[5] http://www.btfusionorder.bt.com/

[6] IEEE 802.15.1. Part 15.1: Wireless Medium Access Control (MAC) and Physical Layer (PHY) Specifications for Wireless Personal Area Networks (WPANs); 2002

[7] http://grouper.ieee.org/groups/802/11/

[8] Punnoose, R.J. Tseng, R.S. Stancil, D.D., "Experimental results for interference between Bluetooth and IEEE 802.11b DSSS systems”, Vehicular Technology Conference, 2001. VTC 2001 Fall. IEEE VTS 54th.

[9] Rosenberg, J., et al., “SIP: Session Initiation Protocol,"RFC 3261, 2002

[10] RFC 3550 - RTP: A Transport Protocol for Real-Time Applications

[11] GSM 04.06, "Digital cellular telecommunications system (Phase 2+); Mobile Station - Base Station System (MS BSS) interface; Data Link (DL) layer specification".

[12] GSM 04.07, "European digital cellular telecommunications system (Phase 2); Mobile radio interface signalling layer 3, General aspects”.

[13] GSM 04.08, "Digital cellular telecommunications system (Phase 2+); Mobile radio interface layer 3 specification”.

[14] GSM 04.11, "Digital cellular telecommunications system (Phase 2+); Point-to-Point (PP) Short Message Service (SMS) support on mobile radio interface".

[15] GSM 03.40, "Digital cellular telecommunications system (Phase 2+); Technical realization of the Short Message Service (SMS); Point-to-Point (PP)”.

[16] SMS Forum Document/Tool Download SMPP Protocol Specification V3.4

[17] GSM 03.22, "Digital cellular telecommunications system (Phase 2+); Functions related to Mobile Station (MS) in idle mode and group receive mode".

[18] GSM 05.08, "Digital cellular telecommunications system (Phase 2+); Radio subsystem link control". 